\title{
Predictors of malaria rapid diagnostic test positivity in a high burden area of Paletwa Township, Chin State in Western Myanmar
}

Pyae Linn Aung ${ }^{1 \dagger}$, Myat Thu Soe ${ }^{1 \dagger}$, Thit Lwin Oo ${ }^{1}$, Aung Khin ${ }^{2}$, Aung Thi ${ }^{3}$, Yan Zhao ${ }^{4}$, Yaming Cao ${ }^{4}$, Liwang Cui ${ }^{5}$, Myat Phone Kyaw ${ }^{1 *}$ and Daniel M. Parker ${ }^{6^{*}}$ (D)

\begin{abstract}
Background: Despite major reductions in malaria burden across Myanmar, clusters of the disease continue to persist in specific subregions. This study aimed to assess the predictors of test positivity among people living in Paletwa Township of Chin State, an area of persistently high malaria burden.

Methods: Four villages with the highest malaria incidence from Paletwa Township were purposively selected. The characteristics of 1045 subjects seeking malaria diagnosis from the four assigned village health volunteers from January to December, 2018 were retrospectively analyzed. Their household conditions and surroundings were also recorded using a checklist. Descriptive statistics and logistic regression models were applied to investigate potential associations between individual and household characteristics and malaria diagnosis.

Results: In 2017, the Paletwa township presented $20.9 \%$ positivity and an annual parasite index of 46.9 cases per 1000 people. Plasmodium falciparum was the predominant species and accounted for more than $80.0 \%$ of all infections. Among 1045 people presenting at a clinic with malaria symptoms, 31.1\% were diagnosed with malaria. Predictors for test positivity included living in a hut [adjusted odds ratios (a OR): $2.3,95 \%$ confidence intervals (C): 1.2-4.6], owning farm animals ( $a O R: 1.7,95 \%$ Cl: 1.1-3.6), using non-septic type of toilets ( $a O R: 1.9,95 \%$ Cl: 1.1-8.4), presenting with fever ( $a O R: 1.9,95 \%$ Cl: 1.1-3.0), having a malaria episode within the last year ( $a O R: 2.9,95 \%$ Cl: 1.4-5.8), traveling outside the village in the previous 14 days (aOR: 4.5, 95\% Cl: 1.5-13.4), and not using bed nets (a OR: 3.4, 95\% Cl: 2.3-5.1). There were no statistically significant differences by age or gender in this present analysis.

Conclusions: The results from this study, including a high proportion of $P$. falciparum infections, little difference in age, sex, or occupation, suggest that malaria is a major burden for these study villages. Targeted health education campaigns should be introduced to strengthen synchronous diagnosis-seeking behaviors, tighten treatment adherence, receiving a diagnosis after traveling to endemic regions, and using bed nets properly. We suggest increased surveillance, early diagnosis, and treatment efforts to control the disease and then to consider the local elimination.
\end{abstract}

Keywords: Malaria, Trend, Risk, Endemic area, Myanmar

*Correspondence: kyaw606@gmail.com; dparker1@hs.uci.edu

${ }^{\dagger}$ Pyae Linn Aung and Myat Thu Soe contributed equally to this work

1 Myanmar Health Network Organization, Yangon, Myanmar

${ }^{6}$ Department of Population Health and Disease Prevention, Department of Epidemiology, University of California, Irvine, USA

Full list of author information is available at the end of the article

\section{Background}

Although the malaria burden has been declining in Myanmar, morbidity and mortality is still high in specific locations, especially in hard-to-reach rural areas. Since 2011, intensive malaria control activities have rapidly expanded by various partners, including the 
National Malaria Control Program (NMCP), with the support of different funding sources [1]. As a result, the country is now striving for malaria elimination, intending to be malaria-free by $2030[2,3]$.

Nevertheless, in 2018, more than 74000 malaria cases were confirmed nationwide [4]. An in-depth analysis revealed that $22.5 \%$ of the total cases came from only 12 endemic townships of five states and regions, namely Chin, Kayin, Rakhine, Kachin, and Sagaing [5]. Thus, there is a relatively unchanged disease burden in some specific areas even though there has been an influx of control and elimination-focused activities. An evaluation of the current interventions, and the piloting of newly produced exercises, were employed.

While remoteness and lack of easy access to diagnosis and treatment may partially account for the high malaria burden in some townships, other environmental factors of communities can also predispose them to higher malaria burdens [6, 7]. Communities located in optimal environments for mosquito vectors may have higher baseline risk than places that are unfavorable for mosquito vectors. The presence of environmental attributes that can attract harmful vectors, such as the ownership of farm animals, non-septic toilets, and living in households favorable for Anopheline mosquitoes, may contribute to a large burden of disease in particular foci [8-10]. Social and behavioral factors can also be important with regard to risk of infection [11-13].

If such environmental, social, or behavioral characteristics are sufficiently predictive of infection, it may be possible to target high-risk subsets of the population (for example migrant populations, adult males, individuals who have recently travelled, etc.) for public health interventions $[14,15]$. Behavioral change communication may be useful for persuading people towards proper prevention practices, especially for use of bed nets or other measures geared toward avoiding exposure to mosquitoes [16]. Conversely, the residents of a village or an area with high transmission may be at equal risk of contracting malaria, regardless of their socio-demographics or behavioral characteristics $[17,18]$. In such scenarios, selectively targeted interventions, rather than implementing setting-specific comprehensive malaria control activities, might be ineffective.

This study was aimed at conducting a detailed epidemiological analysis and assessment of socio-economic factors, for the risk of being diagnosed with malaria in an area with a persistently high burden of this disease (Paletwa Township of Chin State, Myanmar). Few detailed studies have focused on this region. Given the national and regional goals of malaria elimination by 2030, understanding drivers of persistently high burdens in specific areas is important for designing and implementing context specific malaria interventions.

\section{Methods \\ Study design and period}

Three sources of data were used in this study. First, seven consecutive years (2011-2017) of township-wide data from annual reports of the NMCP were tabulated to examine the overall malaria trend and species composition of Paletwa Township. Second, monthly malaria tests and diagnoses from the Myanmar Health Assistant Association (MHAA) during 2017 and 2018 were used to report the total number of febrile patients, total rapid diagnostic tests (RDTs) confirmed cases, and test positivity. Finally, an individual-level analysis was performed using data collected by village health volunteers (VHVs) in four high burden villages in the setting. All resident villagers presented at the malaria clinics in the four study villages between January and December 2018 were included in this study.

\section{Study areas}

The Paletwa Township of Chin State in western Myanmar was purposively selected because it consistently has one of the highest malaria burdens within the country (Fig. 1). The township shares international borders with Bangladesh and India, and also borders Rakhine State. The township has a population of 97053 according to the 2014 nationwide census, the major ethnic group is the Chin, and most inhabitants are farmers.

For malaria control, the township vector borne disease control (VBDC) team and other partners (such as the Myanmar Medical Association, Medical Action Myanmar, Myanmar Council of Churches, and MHAA) are actively involved in malaria control in the township. Based on available data, it was observed that this township had a consistently high malaria prevalence since 2015, when elimination efforts began in Myanmar. In 2018, the township contributed $64.0 \%$ of all malaria cases in Myanmar, with an annual parasite incidence (API) of 15.8/1000 total population at risk (much higher than the targeted pre-elimination goal of API $<1 / 1000$ ).

The MHAA has been operating in this area since late 2016. To explore the macro-level epidemiology of the study township, malaria data from 100 villages covered by the MHAA were collected. The population coverage was approximately $30.0 \%$ of the total population in Paletwa Township.

Next, based on the annual village level data of the MHAA, four villages with the highest malaria test positivity in 2017 were purposively selected to conduct a detailed micro-level epidemiological analysis. All selected high-risk villages were within 7-20 km from the 
central township area, were located along the riverfronts of Kaladan River, and were located near forests (Fig. 1). The easiest means of transportation from these villages to the nearest large town area is by the river via a boat or shuttle. The total population of the four study villages was 2404 (MeeLatwa village is the largest).

\section{Data collection and malaria diagnoses}

All residents were encouraged to have a blood test soon after showing signs of fever and to strengthen proper preventive measures, especially the use of insecticidetreated nets. For the malaria diagnosis, RDTs detecting $P$. falciparum and $P$. vivax antigens were utilized (SD BIOLINE Malaria Ag Pf/Pv) by VHVs.

The assigned VHV delivered the malaria diagnosis and standard treatment to each suspected patient and recorded the data in a carbonless case report form (CRF). Each CRF addressed the general characteristics of individuals including age in years, sex, presence or absence of fever at the time of diagnosis, RDT result either $P$. falciparum or $P$. vivax or both, and treatment administered. The presence or absence of fever was reported on the basis of the patients' responses through proper history taking by the VHV. In addition, the VHVs were requested to complete an extra checklist for each febrile patient addressing occupation, education level, travel history of patients, and history concerning malaria attacks, which were not included in the current official CRFs. The occupations of study participants were categorized into the forest or non-forest related types. Goldminers, farmers, and lumberjacks were defined as forest related occupations while government officers, monks, and merchants were represented as non-forest related types of occupation.

Other factors regarding household conditions and the environmental situations were observed and recorded by the study team using a checklist after a person came to the clinic. VHVs visited the patients' households and each household's leader was directly asked about the conditions of their households and toilets, and the presence of pigs or cattle during the study year. Households were broadly categorized as hut, manor, or cottage; and toilets were broadly categorized as: non-septic type (pit latrine) or septic type (septic tank or pour-flush latrine) (Additional file 1: Additional figures). In the case that house or toilet conditions changed during the study period, the prevailing conditions were taken into account. Only residents of the study villages were included in this study.

Data assurance and data quality were thoroughly and closely checked by the study team and updated as necessary. The forms were then collected and data were entered in a formatted Microsoft Excel (Excel for Mac,
Version 16.16.27, Seattle, USA) spreadsheet using a password protected-computer on a case-by-case basis.

\section{Data analysis}

RDT positivity and API were estimated and plotted for the 100 villages of Paletwa Township by year. Data from the four targeted high-burden villages (JanuaryDecember 2018) were plotted by month. Summary and descriptive statistics were used to assess the correlates of RDT positivity for febrile patients in the four study villages. Logistic regression (both bi- and multi-variable) was used to investigate potential predictors of RDT positivity among febrile patients in the four study villages. Individuals with repeat episodes were counted only once. All logistic regression results are presented as model adjusted odds ratios (a OR) with 95\% confidence intervals $(C I)$. P-values for summary statistics were adjusted using the Bonferroni approach. All analyses were conducted using the Statistical Package for the Social Sciences (IBM SPSS Statistics for Macintosh, Version 23, IBM Corp., Armonk, USA).

\section{Ethics consideration}

The protocol for this study was examined and approved by the institutional review board, Department of Medical Research (Lower Myanmar), and from the University of South Florida, USA.

\section{Results}

Malaria trends in Paletwa Township from 2011 through 2017

In Paletwa, there was a general reduction of API from $86.8 / 1000$ in 2011 to $46.9 / 1000$ population in 2017 (Fig. 2a). However, the 2017 API in Paletwa was almost 30 times higher than the nationwide API of 1.6. It is noteworthy that the trend was irregular, and the highest API was observed in 2015, when the country started the malaria elimination era and encouraged malaria surveillance system to detect more suspected patients. Similarly, RDT positivity declined progressively each year. While $54.6 \%$ of all tests were positive in 2011, 20.9\% were positive in 2017 (Fig. 2a). P. falciparum was the dominant species reported in Paletwa Township. Among the total 48144 infections during 2011-2017, almost 88.0\% were infected by $P$. falciparum. In each year, $P$. falciparum accounted more than $80.0 \%$ of total infections (Fig. 2b). The relative contribution of $P$. vivax to the overall burden of malaria appears to increase over time.

\section{Malaria trends and seasonality in four high burden study villages}

During the study period (January-December 2018), 1045 participants had blood tests by RDTs from four 


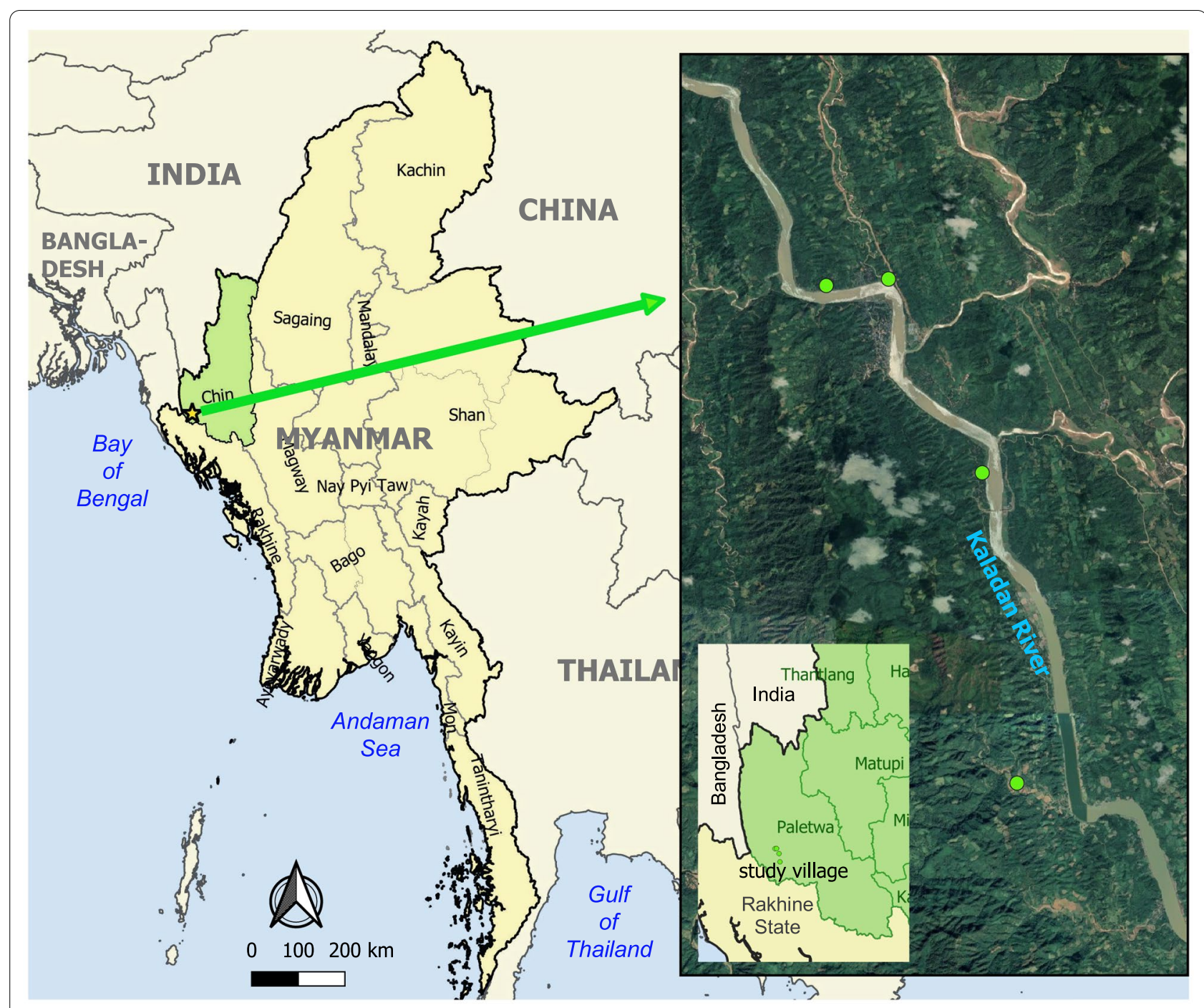

Fig. 1 Map indicating study location in Paletwa Township, Chin State, Western Myanmar

assigned VHVs, and all were eligible to participate in this study. Among them, 325 patients were diagnosed with malaria infections (31.1\%). There were five participants with repeat infections during the study period (four with repeated $P$. falciparum episodes and one with a repeat $P$. vivax episode). In the absence of molecular data on the parasite strains, it is unclear whether these were new infections.

RDT positivity in the four study villages declined from $29.1 \%$ in 2017 to $24.0 \%$ in 2018 . Most infections were from P. falciparum (73.1\%), followed by P. vivax (25.0\%). Mixed infections accounted for $1.9 \%$ of all diagnosed malaria infections. A small increase in the proportion of P. vivax cases was observed in 2017 (Table 1).

During the analysis of monthly malaria data for 20172018, the disease incidence showed a typical seasonal pattern during the two years. The number of cases was highest in June and lowest in March for both the years. A second peak was also observed in December 2017. The number of diagnoses was the highest at the onset of the rainy season (June-October) and steeply declined after the cold season (November-January) and during the dry season (February-April). Similar fluctuations were observed in RDT positivity (Fig. 3) and total febrile cases.

\section{Socio-demographic characteristics and risks factors}

Most of the febrile patients were below the age of 25 years old (63.2\%), and male (53.4\%); $53.0 \%$ were unemployed (including children, students, and housewives), and $44.4 \%$ had forest-related occupations. Few had above primary education (19.0\%). The majority of participants lived in huts (93.5\%), 94.4\% had farm animals (either pig or cattle or both), and $91.2 \%$ reported having a non-septic 
toilet at their home (Table 2 and Additional file 1: Figure S1). A relatively high proportion (32.2\%) reported not using bed-nets. Most of the patients (72.3\%) presented with fever, few reported having a malaria episode during the last year (6.2\%), and $2.6 \%$ reported traveling outside of the village within one month before coming to undergo a blood test.

\section{Predictors of being diagnosed with malaria}

No statistically significant associations were found between malaria diagnosis and age, sex, occupation, and education level of the study subjects. Patients who presented with fever, reported previous malaria episodes, or travelled to outside villages, reported not using bed-nets were more likely to be diagnosed with malaria $(P<0.001)$. Individuals living in huts, especially those with latrinetype toilets, and having livestock were also more likely to be diagnosed with malaria $(P<0.001)$ (Table 3$)$.

Both simple logistic and multi-variable logistic regression analyses revealed similar results (Table 4). The multi-variable logistic regression indicated statistically significant associations between presenting with fever [a $O R$ and $95 \%$ CI of $1.9(1.1-3.0)$ ], reporting previous malaria attacks $[2.9(1.4-5.8)]$ and reporting traveling outside their villages in the previous month [4.5 (1.513.4)]. In addition, participants who reported not using bed-nets had 3.4 times the odds of being diagnosed with malaria than those who reported using bed nets $(95 \% \mathrm{CI}$ 2.3-5.1). Moreover, there were higher odds of malaria infection among some socio-economic characteristics, including: participants living in huts [2.3 (1.2-4.6)], having farm animals $[1.7(1.1-3.6)]$ and using latrine-type toilets $[1.9(1.1-8.4)]$.

\section{Discussion}

The overall malaria positivity in the study reported by MHAA during 2018 was 24.0\% (4075 confirmed cases/16 959 total febrile patients). Although Paletwa Township consistently shows a high malaria burden, the situation is heterogeneous, with some villages contributing more to the overall malaria burden than others do. Implementation strategies will benefit from
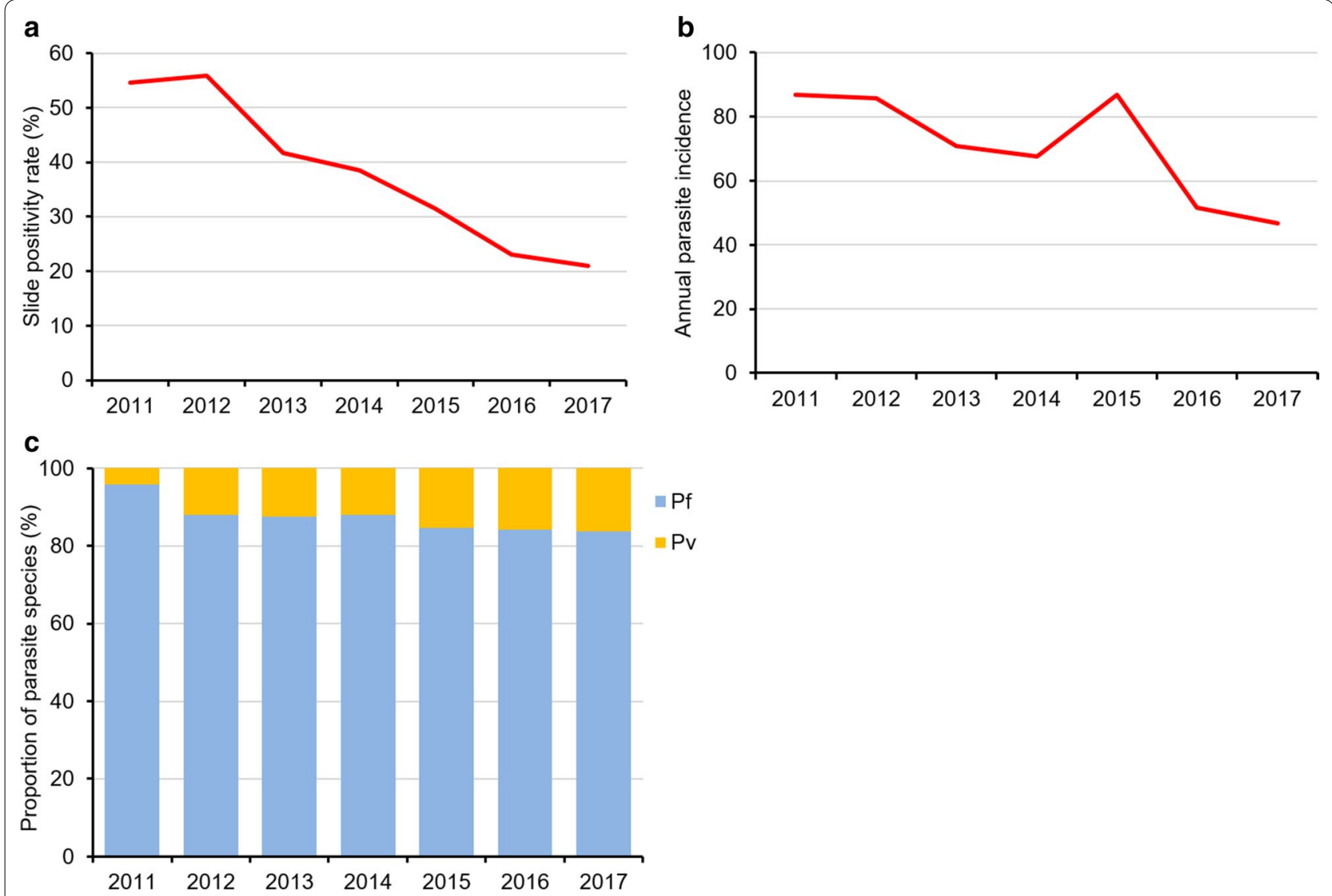

Fig. 2 Township level malaria data for Paletwa: a Test positivity and $\mathbf{b}$ annual parasite incidence (2011-2017). c Plasmodium species proportions (2011-2017). Pf: Plasmodium falciparum; Pv: Plasmodium vivax 
in-depth village-level microstratification of targeted townships whenever possible.

For $P$. falciparum infections, the likelihood of onward transmission decreases when symptomatic individuals quickly receive treatment (as gametocytes take time to develop) [19]. In this study area, therefore, enhanced early diagnosis together with effective treatment compliance, especially by gametocidal and hypnozoiticidal drugs, are needed to interrupt onward locally acquired transmission [20]. The apparent increase in the contribution of $P$. vivax to the overall malaria burden may indicate that diagnosis and treatment efforts have an

Table 1 Malaria situation in Paletwa Township (20172018)

\begin{tabular}{llll}
\hline Descriptions & $\mathbf{2 0 1 7}$ & $\mathbf{2 0 1 8}$ & Total \\
\hline Malaria positivity & & & \\
Total febrile cases tested & 17520 & 16959 & 34479 \\
$\begin{array}{l}\text { Confirmed cases } \\
\text { Positivity rate (\%) }\end{array}$ & 5106 & 4075 & 9181 \\
$\begin{array}{l}\text { Malaria parasite [n (\%)] } \\
\text { Plasmodium falciparum }\end{array}$ & $3981(78.0)$ & $2738(67.2)$ & $6719(73.1)$ \\
$\begin{array}{l}\text { P. vivax } \\
\text { Mixed infections }\end{array}$ & $1020(20.0)$ & $1272(31.2)$ & $2292(25.0)$ \\
& $105(2.0)$ & $65(1.6)$ & $170(1.9)$ \\
\hline
\end{tabular}

The data reflected only for 100 implemented villages by Myanmar Health Assistant Association impact already. Targeted health education campaigns may also improve treatment seeking behaviors among community members [21]. Furthermore, to increase access to early diagnosis and treatment in this remote area, increased implementation of the VHV workforce would be a key activity as suggested in a study from eastern Myanmar [22]. Regular active case detection, either by mobile clinics or diagnosis centers, might also be advantageous for the early detection of disease and to cover the gaps in passive case detection [23, 24]. Interventions such as mass drug administration should only be considered in this setting when a profound surveillance system and proper vector control measures are in place [25].

Among the study participants in the four study villages presented with fever, $34.5 \%$ showed positive infections. Fever associated with chill and rigor was seen in more than $90.0 \%$ of patients with malaria [26]. In Myanmar, many people seek treatment from unlicensed healers or quacks, potentially leading to onward transmission of the disease and poor health outcomes for infected individuals. Strengthening legislation or introducing methods to improve people's health understanding and training should be considered to address this problem [27]. Asymptomatic parasite carriers could continue to frustrate control efforts even in the presence of easily accessible diagnosis and treatment

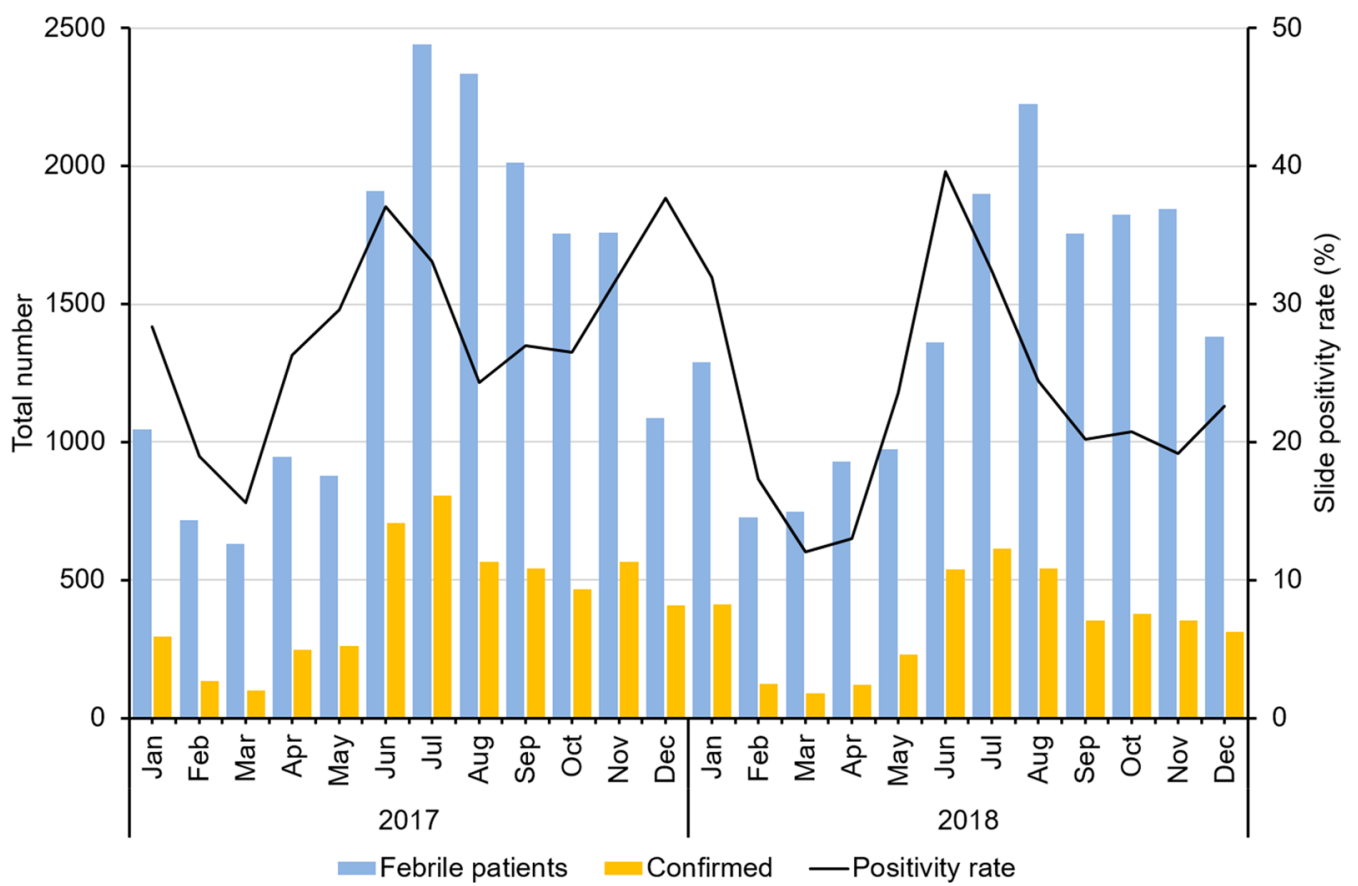

Fig. 3 Malaria cases in 2017 and 2018 in the four study villages (including total febrile patients, total confirmed cases, and test positivity) 
Table 2 Descriptive statistics for study participants $(n=1045)$ from four study villages in Paletwa Township, Chin State, Myanmar (January-December 2018)

\begin{tabular}{|c|c|c|}
\hline Description & Frequency $(n)$ & Percentage (\%) \\
\hline \multicolumn{3}{|l|}{ Malaria infection found by RDT } \\
\hline Positive cases & 325 & 31.1 \\
\hline Plasmodium falciparum $(n=325)$ & 251 & 77.2 \\
\hline P. vivax $(n=325)$ & 41 & 12.6 \\
\hline Mixed $(n=325)$ & 33 & 10.2 \\
\hline Negative cases & 720 & 68.9 \\
\hline \multicolumn{3}{|c|}{ Population ( $n=2404)$ and malaria cases distribution } \\
\hline LaungKaDuu village $(n=674)$ & 83 & 25.5 \\
\hline MeeLatWa village $(n=894)$ & 113 & 34.8 \\
\hline MonDaungChaungHtel village $(n=364)$ & 64 & 19.7 \\
\hline YokeWa village $(n=472)$ & 65 & 20.0 \\
\hline \multicolumn{3}{|l|}{ Age (years) } \\
\hline$<5$ & 184 & 17.6 \\
\hline $5-14$ & 248 & 23.7 \\
\hline $15-24$ & 229 & 21.9 \\
\hline$\geq 25$ & 384 & 36.8 \\
\hline \multicolumn{3}{|l|}{ Sex } \\
\hline Male & 558 & 53.4 \\
\hline Female & 487 & 46.6 \\
\hline \multicolumn{3}{|l|}{ Occupation } \\
\hline Unemployed & 554 & 53.0 \\
\hline Forest related workers & 464 & 44.4 \\
\hline Non-forest related workers & 27 & 2.6 \\
\hline \multicolumn{3}{|l|}{ Education level } \\
\hline$\leq$ Primary education & 846 & 81.0 \\
\hline > Primary education & 199 & 19.0 \\
\hline \multicolumn{3}{|l|}{ Type of household } \\
\hline Hut & 977 & 93.5 \\
\hline Others & 68 & 6.5 \\
\hline \multicolumn{3}{|l|}{ Having farm animals (cattle/pigs) } \\
\hline Yes & 987 & 94.4 \\
\hline \multicolumn{3}{|l|}{ Type of toilet } \\
\hline Non-septic & 953 & 91.2 \\
\hline Septic & 92 & 8.8 \\
\hline
\end{tabular}

$R D T$ rapid diagnostic test

[28]. Targeted approaches may be necessary to address asymptomatic reservoirs.

Participants with a history of malaria within the last year had 2.9 times the odds of being diagnosed with malaria than those without a history. One reason for this is that these people may have a forest-related job or live in an environment close to vector breeding sites, making them vulnerable to repeated malaria infections. Also, as Myanmar has many threats to universal health care coverage, some people in this remote area might not be able to use preventive measures such as bed nets or treatment services [29]. Additionally, some recurrent episodes may be the result of relapses or recrudescence (for P. vivax infections) [30]. Further, detailed analyses should assess the causes of repeated malaria infections in one person in one single year should also be conducted with follow-up actions taken accordingly.

Traveling outside the village was also a significant predictor of being diagnosed with malaria. While the majority of participants reported using bed-nets, many also reported that when they went outside the village, especially to work, protective measures were neglected [31]. A similar situation was also found at the Thai-Myanmar border, where residual malaria transmission is closely 
Table 3 Summary statistics for potential predictors of malaria test positivity among study participants ( $n=1045$ ) from four study villages in Paletwa Township, Chin State, Myanmar (January-December 2018)

\begin{tabular}{|c|c|c|c|}
\hline \multirow[t]{3}{*}{ Description } & \multicolumn{2}{|c|}{ Malaria infection } & \multirow[t]{3}{*}{$P$-value } \\
\hline & Positive & Negative & \\
\hline & (\%) & (\%) & \\
\hline
\end{tabular}

\begin{tabular}{|c|c|c|c|c|c|}
\hline \multicolumn{6}{|l|}{ Age (years) } \\
\hline$<5$ & 63 & 34.2 & 121 & 65.8 & \multirow[t]{4}{*}{0.780} \\
\hline $5-14$ & 77 & 31.0 & 171 & 69.0 & \\
\hline $15-24$ & 69 & 30.1 & 160 & 69.9 & \\
\hline$\geq 25$ & 116 & 30.2 & 268 & 69.8 & \\
\hline \multicolumn{6}{|l|}{ Sex } \\
\hline Male & 185 & 33.2 & 373 & 66.8 & \multirow[t]{2}{*}{0.273} \\
\hline Female & 140 & 28.7 & 347 & 71.3 & \\
\hline \multicolumn{6}{|l|}{ Occupation ${ }^{* *}$} \\
\hline Unemployed & 183 & 33.0 & 371 & 67.0 & \multirow[t]{3}{*}{0.519} \\
\hline Forest related workers & 133 & 28.7 & 331 & 71.3 & \\
\hline Non-forest related workers & 9 & 33.3 & 18 & 66.7 & \\
\hline \multicolumn{6}{|l|}{ Education level } \\
\hline$\leq$ Primary education & 249 & 29.4 & 597 & 70.6 & \multirow[t]{2}{*}{0.293} \\
\hline > Primary education & 76 & 38.2 & 123 & 61.8 & \\
\hline \multicolumn{6}{|l|}{ Type of household } \\
\hline Hut & 282 & 28.9 & 695 & 71.1 & \multirow[t]{2}{*}{$<0.001^{*}$} \\
\hline Others & 43 & 63.2 & 25 & 36.8 & \\
\hline \multicolumn{6}{|c|}{ Having farm animals (cattle/pigs) } \\
\hline Yes & 287 & 29.1 & 700 & 70.9 & \multirow[t]{2}{*}{$<0.001^{*}$} \\
\hline No & 38 & 65.5 & 20 & 34.5 & \\
\hline \multicolumn{6}{|l|}{ Type of toilet } \\
\hline Non-septic & 266 & 27.9 & 687 & 72.1 & \multirow[t]{2}{*}{$<0.001^{*}$} \\
\hline Septic & 59 & 64.1 & 33 & 35.9 & \\
\hline \multicolumn{6}{|l|}{ Having fever } \\
\hline Yes & 261 & 34.5 & 495 & 65.5 & \multirow[t]{2}{*}{$0.005^{*}$} \\
\hline No & 64 & 22.1 & 225 & 77.9 & \\
\hline \multicolumn{6}{|c|}{ Having episode of malaria in last year } \\
\hline Yes & 40 & 61.5 & 25 & 38.5 & \multirow[t]{2}{*}{$<0.001^{*}$} \\
\hline No & 285 & 29.1 & 695 & 70.9 & \\
\hline \multicolumn{6}{|c|}{ Traveling to malaria endemic area of outside the village } \\
\hline Yes & 19 & 70.4 & 8 & 29.6 & \multirow[t]{2}{*}{$0.001^{*}$} \\
\hline No & 306 & 30.1 & 712 & 69.9 & \\
\hline \multicolumn{6}{|l|}{ Use of bed nets } \\
\hline Yes & 154 & 21.8 & 554 & 78.2 & \multirow[t]{2}{*}{$<0.001^{*}$} \\
\hline No & 171 & 50.7 & 166 & 49.3 & \\
\hline
\end{tabular}

Degree of freedom for Chi-square test $=1$, ${ }^{* *}$ Fisher exact test, ${ }^{*} P<0.005$, using the Bonferroni correction for multiple tests

tied to temporary farm huts or shelters during the agricultural season, when people travel to these sites and stay overnight without proper protection [32]. Increasing bed net use and distributing other protective gear (such
Table 4 Logistic regression models to explore the associations between dependent variables and proneness to have malaria infection $(n=1045)$

\begin{tabular}{|c|c|c|c|c|}
\hline Description & $\mathrm{COR}$ & $95 \% \mathrm{Cl}$ & $\mathrm{aOR}$ & $95 \% \mathrm{Cl}$ \\
\hline \multicolumn{5}{|c|}{ Type of household } \\
\hline Others & 1.0 & & 1.0 & \\
\hline Hut & 3.2 & $(1.6-7.4)$ & 2.3 & $(1.2-4.6)$ \\
\hline \multicolumn{5}{|c|}{ Having farm animals (cattle/pigs) } \\
\hline Yes & 2.5 & $(1.4-5.4)$ & 1.7 & $(1.1-3.6)$ \\
\hline No & 1.0 & & 1.0 & \\
\hline \multicolumn{5}{|l|}{ Type of toilet } \\
\hline Septic & 1.0 & & 1.0 & \\
\hline Non-septic & 3.3 & $(2.1-9.4)$ & 1.9 & $(1.1-8.4)$ \\
\hline \multicolumn{5}{|l|}{ Having fever } \\
\hline Yes & 1.9 & $(1.2-2.9)$ & 1.9 & $(1.1-3.0)$ \\
\hline No & 1.0 & & 1.0 & \\
\hline \multicolumn{5}{|c|}{ Having episode of malaria in last year } \\
\hline Yes & 3.8 & $(2.1-7.1)$ & 2.9 & $(1.4-5.8)$ \\
\hline No & 1.0 & & 1.0 & \\
\hline \multicolumn{5}{|c|}{ Traveling to malaria endemic area of outside the village } \\
\hline Yes & 5.5 & $(2.1-14.2)$ & 4.5 & $(1.5-13.4)$ \\
\hline No & 1.0 & & 1.0 & \\
\hline \multicolumn{5}{|c|}{ Use of bed nets } \\
\hline Yes & 1.0 & & 1.0 & \\
\hline No & 3.7 & $(2.5-5.3)$ & 3.4 & $(2.3-5.1)$ \\
\hline
\end{tabular}

95\% Cl: 95\% confidence interval, cOR: crude odd ratio by simple logistic regression, aOR: adjusted odd ratio by multiple logistic regression

as insecticide-treated clothes and mosquito repellents) may help address this problem. Diagnosis and treatment efforts that focus on people returning from outside the village, or on people visiting the village, could potentially catch imported infections.

Several studies $[18,33,34]$ have shown protective effects of using bed nets. In this study approximately $30 \%$ of participants reported not using any nets. Research that focuses on the reasons why people were not using bed nets is warranted. Studies from other regions have illustrated a variety of factors that influence bed net use [35-37]. Procurement policies should account for people's preferences in addition to other technical aspects of insecticide efficacy and cost-effectiveness.

Household construction and conditions (including the type of toilet and livestock ownership) were associated with malaria infections in this study. Several other studies have shown similar results, in a variety of settings [38-41]. Further work on the ecology of malaria vectors in this region may help to explain this association better. One vector study [42] in Myanmar reported that in the study township (Paletwa), Anopheles minimus served as the major vector. Adults preferred to rest in houses, were anthropophilic and fed beginning at dusk with a 
peak around midnight (both outdoors and indoors) and a gradual decrease in feeding activity toward the dawn (6:00 am). An. maculatus, An. sinensis, An. philippinensis, and $A n$. annularis were suggested to be secondary vectors, feeding both humans and animals. Interventions that consider household design and construction may therefore be warranted.

Several studies from the Greater Mekong Subregion have shown that $P$. falciparum infections are frequently acquired among adult males [43, 44]. In the current study, however, the respondents' age was inconsequential to the risk of being diagnosed with malaria. This finding corresponds to one from areas with a high malaria burden in eastern Myanmar [45]. Once active foci are eliminated through extensive malaria control measures (including expansion of access to diagnosis and treatment facility), $P$. falciparum transmission persists in remote areas away from the village-becoming an occupational hazard. Subsequently, $P$. falciparum cases are mostly observed among adult males who were probably infected from somewhere else outside the village [46].

This study has some limitations. Some villagers may have been diagnosed and treated elsewhere and would not have been detected in this study. The presence of malaria infection was confirmed only by RDTs as the study area had no access to microscopy examination. There may be asymptomatic infections in the community, which are unlikely to be detected by the VHV. In order to understand the total malaria burden in this setting it would be valuable to conduct a prevalence survey with highly sensitive screening tests. Febrile illnesses that were not diagnosed as malaria did not receive final diagnoses, as the VHVs are limited in their diagnostic capabilities. Further work that includes more detailed and longitudinal assessments of the predictors of malaria infections in this region are warranted.

\section{Conclusions}

The results of this study suggested potential risk factors to be aware of in preventing malaria infection among people living in this high burden area. People living in huts, with non-septic toilets, possessing farm animals, presenting with fever, having a malaria episode in the last year, traveling to any outside village in the last 14 days and not using bed nets were more likely to have a positive malaria test by RDT. In addition to current exclusive malaria control activities, modifying these factors may also reduce caseloads. To improve access to malaria diagnosis and treatment, services should also be encouraged by expanding the VHV workforce. Furthermore, a large proportion of febrile illnesses were not diagnosed as malaria. The causative agent behind these illnesses normally remains unknown. As these illnesses are likely to remain while the malaria burden decreases, it is important to address these non-malarial febrile illnesses. Targeted health education campaigns should be introduced to strengthen synchronous diagnosis-seeking behaviors, tighten treatment adherence, receiving a diagnosis after traveling to endemic regions, and using bed nets properly. On the other hand, the economic index of a community should be improved by collaborating with local government agencies, nonstate actors, and other community development organizations. Finally, special attention should be paid to this and other high burden areas so that they do not frustrate national elimination efforts.

\section{Supplementary Information}

The online version contains supplementary material available at https://doi. org/10.1186/s40249-020-00787-z.

Additional file 1: Additional figures.

Acknowledgements

We thank all village health volunteers for their commitment to the health of their communities and for data collection for this research.

\section{Authors' contributions}

PLA, MTS, LC, MPK and DMP conceived the design of this study. TLO, AK, AT and MPK facilitated data collection. PLA, LC and DMP performed analysis, and drafted the manuscript. All authors have reviewed and revised the manuscript. All authors read and approved the final manuscript.

\section{Funding}

This research was financially supported a grant (U19AI089672) from the National Institute of Allergy and Infectious Diseases, National Institutes of Health, USA

\section{Availability of data and materials}

All data analyzed for this study are included within the article.

\section{Ethics approval and consent to participate}

This study was reviewed and approved by the Institutional Review Board of Department of Medical Research, Myanmar and the University of South Florida.

\section{Consent for publication}

Not applicable.

\section{Competing interests}

The authors declare that they have no conflict of interests.

\section{Author details}

${ }^{1}$ Myanmar Health Network Organization, Yangon, Myanmar. ${ }^{2}$ Myanmar Health Assistant Association, Yangon, Myanmar. ${ }^{3}$ Department of Public Health, Ministry of Health and Sports, NayPyiTaw, Myanmar. ${ }^{4}$ Department of Immunology, College of Basic Medical Science, China Medical University, Shenyang 110122, Liaoning, China. ${ }^{5}$ Division of Infectious Diseases and International Medicine, Department of Internal Medicine, Morsani College of Medicine, University of South Florida, 3720 Spectrum Boulevard, Suite 304, Tampa, FL 33612, USA. ${ }^{6}$ Department of Population Health and Disease Prevention, Department of Epidemiology, University of California, Irvine, USA.

Received: 31 July 2020 Accepted: 16 December 2020

Published online: 11 January 2021 


\section{References}

1. Ministry of Health and Sports. National strategic plan: intensifying malaria control and accelerating progress towards malaria elimination 2016-2020. Myanmar: Department of Public Health; 2017 a.

2. World Health Organization. Strategy for malaria elimination in the greater Mekong Sub region (2015-2030). Geneva: WHO; 2015a.

3. Ministry of Health and Sports. National plan for malaria elimination in Myanmar 2016-2030. Myanmar: Department of Public Health; 2017b.

4. World Health Organization. World malaria report 2019. Geneva: WHO; 2019.

5. Program NMC. VBDC annual report 2018. Myanmar: Deaprtment of Public Health; 2019

6. Kibret S, Glenn Wilson G, Ryder D, Tekie H, Petros B. Environmental and meteorological factors linked to malaria transmission around large dams at three ecological settings in Ethiopia. Malar J. 2019;18(1):54.

7. Rossati A, Bargiacchi O, Kroumova V, Zaramella M, Caputo A, Garavelli PL. Climate, environment and transmission of malaria. Infez Med. 2016;24(2):93-104.

8. Mayagaya VS, Nkwengulila G, Lyimo IN, Kihonda J, Mtambala H, Ngonyani $\mathrm{H}$, et al. The impact of livestock on the abundance, resting behaviour and sporozoite rate of malaria vectors in southern Tanzania. Malar J. 2015;14(1):17.

9. Nakagiri A, Niwagaba CB, Nyenje PM, Kulabako RN, Tumuhairwe JB, Kansiime F. Are pit latrines in urban areas of Sub-Saharan Africa performing? A review of usage, filling, insects and odour nuisances. BMC Public Health. 2016;16(1):120.

10. Mzilahowa T, Luka-Banda M, Uzalili V, Mathanga DP, Campbell CH Jr, Mukaka M, et al. Risk factors for Anopheles mosquitoes in rural and urban areas of Blantyre District, southern Malawi. Malawi Med J. 2016;28(4):154-8

11. Hasyim H, Dale P, Groneberg DA, Kuch U, Muller R. Social determinants of malaria in an endemic area of Indonesia. Malar J. 2019;18(1):134.

12. Houmsou RS, Amuta EU, SarTT, Adagba AH. Malarial infection among patients attending a Nigerian semi-urban based hospital and performance of HRP-2 pf Rapid diagnostic Test (RDT) in screening clinical cases of Plasmodium falciparum malaria. Translational Biomed. 2011;2(1):5.

13. Ricci F. Social implications of malaria and their relationships with poverty. Mediterr J Hematol Infect Dis. 2012:4(1):e2012048.

14. Nguitragool W, Karl S, White M, Koepfli C, Felger I, Singhasivanon P, et al. Highly heterogeneous residual malaria risk in western Thailand. Int J Parasitol. 2019;49(6):455-62.

15. Bartoloni A, Zammarchi L. Clinical aspects of uncomplicated and severe malaria. Mediterr J Hematol Infect Dis. 2012;4(1):e2012026.

16. Kilian A, Lawford H, Ujuju CN, Abeku TA, Nwokolo E, Okoh F, et al. The impact of behaviour change communication on the use of insecticide treated nets: a secondary analysis of ten post-campaign surveys from Nigeria. Malar J. 2016;15(1):422.

17. Cook J, Owaga C, Marube E, Baidjoe A, Stresman G, Migiro R, et al. Risk factors for Plasmodium falciparum infection in the Kenyan Highlands: a cohort study. Trans R Soc Trop Med Hyg. 2019;113(3):152-9.

18. World Health Organization. A framework for malaria elimination 2016-2030. Geneva:WHO; 2017.

19. Drakeley C, Sutherland C, Bousema JT, Sauerwein RW, Targett GA. The epidemiology of Plasmodium falciparum gametocytes: weapons of mass dispersion. Trends Parasitol. 2006;22(9):424-30.

20. Landier J, Parker DM, Thu AM, Carrara VI, Lwin KM, Bonnington CA, et al. The role of early detection and treatment in malaria elimination. Malar J. 2016:15:363.

21. Vilay P, Nonaka D, Senamonty P, Lao M, Iwagami M, Kobayashi J, et al. Malaria prevalence, knowledge, perception, preventive and treatment behavior among military in Champasak and Attapeu provinces, Lao PDR: a mixed methods study. Trop Med Health. 2019;47:11.

22. Landier J, Parker DM, Thu AM, Lwin KM, Delmas G, Nosten FH, et al. Effect of generalised access to early diagnosis and treatment and targeted mass drug administration on Plasmodium falciparum malaria in Eastern Myanmar: an observational study of a regional elimination programme. Lancet. 2018;391(10133):1916-26.

23. Smith Gueye C, Sanders KC, Galappaththy GN, Rundi C, Tobgay T, Sovannaroth $\mathrm{S}$, et al. Active case detection for malaria elimination: a survey among Asia Pacific countries. Malar J. 2013;12(1):358.
24. Wickremasinghe R, Fernando SD, Thillekaratne J, Wijeyaratne PM, Wickremasinghe AR. Importance of active case detection in a malaria elimination programme. Malar J. 2014;13(5):186

25. World Health Organization. The role of mass drug administration, mass screening and treatment, and focal screening and treatment for malaria. Geneva: WHO; 2015b

26. Jelia S, Meena S, Meena SR, et al. A study of clinical profile and complication of malaria in a tertiary care centre in South-eastern region of Rajasthan, India. Int J Adv Med. 2016;3(3):614.

27. Sudhinaraset M, Briegleb C, Aung M, Khin HS, Aung T. Motivation and challenges for use of malaria rapid diagnostic tests among informal providers in Myanmar: a qualitative study. Malar J. 2015;14(2):61.

28. Chaumeau V, Kajeechiwa L, Fustec B, Landier J, Naw Nyo S, Nay Hsel S, et al. Contribution of asymptomatic Plasmodium infections to the transmission of malaria in Kayin State. Myanmar J Infect Dis. 2019;219(9):1499-509.

29. Latt NN, Myat Cho S, Htun NM, Yu Mon S, Myint MN, Aoki F, et al. Healthcare in Myanmar. Nagoya J Med Sci. 2016;78(2):123-34.

30. Bisoffi Z, Gobbi F, Buonfrate D, Van den Ende J. Diagnosis of malaria infection with or without disease. Mediterr J Hematol Infect Dis. 2012;4(1):e2012036

31. Stewart T, RP M. Factors that affect the success and failure of Insecticide Treated Net Programs for malaria control in SE Asia and the Western Pacific. Geneva: World Health Organization; 2003.

32. Edwards HM, Sriwichai P, Kirabittir K, Prachumsri J, Chavez IF, Hii J. Transmission risk beyond the village: entomological and human factors contributing to residual malaria transmission in an area approaching malaria elimination on the Thailand-Myanmar border. Malar J. 2019;18(1):221.

33. Ntonifor $\mathrm{NH}$, Veyufambom $\mathrm{S}$. Assessing the effective use of mosquito nets in the prevention of malaria in some parts of Mezam division, Northwest Region Cameroon. Malar J. 2016;15(1):390.

34. Nyamai R, Knight V, Ochanda D. Effective utilization of insecticide treated nets and hospitalization of children under five years in Matete health center in western Kenya. Int J Dev Res. 2018;8(3):4.

35. Pulford J, Hetzel MW, Bryant M, Siba PM, Mueller I. Reported reasons for not using a mosquito net when one is available: a review of the published literature. Malar J. 2011;11(4):83.

36. Gobena T, Berhane Y, Worku A. Low long-lasting insecticide nets (LLINs) use among household members for protection against mosquito bite in Kersa, Eastern Ethiopia. BMC Public Health. 2012;12(12):914.

37. Taremwa IM, Ashaba S, Adrama HO, Ayebazibwe C, Omoding D, Kemeza I, et al. Knowledge, attitude and behaviour towards the use of insecticide treated mosquito nets among pregnant women and children in rural Southwestern Uganda. BMC Public Health. 2017;17(1):794.

38. Ondiba IM, Oyieke FA, Ong'amo GO, Olumula MM, Nyamongo IK, Estambale BBA. Malaria vector abundance is associated with house structures in Baringo County, Kenya. PLoS One. 2018;13(6):e0198970.

39. Fobil JN, Kraemer A, Meyer CG, May J. Neighborhood urban environmental quality conditions are likely to drive malaria and diarrhea mortality in Accra, Ghana. J Environ Public Health. 2011;2011:484010.

40. Hiscox A, Khammanithong P, Kaul S, Sananikhom P, Luthi R, Hill N, et al. Risk factors for mosquito house entry in the Lao PDR. PLoS One. 2013:8(5):e62769

41. Charlwood JD, Pinto J, Ferrara PR, Sousa CA, Ferreira C, Gil V, et al. Raised houses reduce mosquito bites. Malar J. 2003:2(1):45.

42. Ool TT, Storch V, Becker N. Review of the anopheline mosquitoes of Myanmar. J Vector Ecol. 2004;29(1):21-40.

43. Wang Y, Yang Z, Yuan L, Zhou G, Parker D, Lee MC, et al. Clinical efficacy of dihydroartemisinin-piperaquine for the treatment of uncomplicated Plasmodium falciparum malaria at the China-Myanmar border. Am J Trop Med Hyg. 2015;93(3):577-83.

44. Aung T, Wei C, McFarland W, Aung YK, Khin HS. Ownership and use of insecticide-treated nets among people living in malaria endemic areas of eastern Myanmar. PLoS One. 2016:11(9):e0162292.

45. Parker DM, Matthews SA, Yan G, Zhou G, Lee MC, Sirichaisinthop J, et al. Microgeography and molecular epidemiology of malaria at the ThailandMyanmar border in the malaria pre-elimination phase. Malar J. 2015;14:198.

46. Kigozi SP, Kigozi RN, Epstein A, Mpimbaza A, Sserwanga A, Yeka A, et al. Rapid shifts in the age-specific burden of malaria following successful control interventions in four regions of Uganda. Malar J. 2020;19(1):128. 